\title{
Proteomic analysis of human cervicovaginal fluid collected before preterm premature rupture of the fetal membranes
}

\author{
Stella Liong ${ }^{1,2}$, Megan K W Di Quinzio ${ }^{1,2}$, Yujing J Heng ${ }^{3}$, Gabrielle Fleming², \\ Michael Permezel ${ }^{1,2}$, Gregory E Rice ${ }^{4}$ and Harry M Georgiou ${ }^{1,2}$ \\ ${ }^{1}$ Department of Obstetrics and Gynaecology, University of Melbourne, Parkville, Victoria, Australia, ${ }^{2}$ Mercy Perinatal \\ Research Centre, Mercy Hospital for Women, Heidelberg, Victoria, Australia, ${ }^{3}$ Samuel Lunenfeld Research Institute, \\ Research Centre for Women's and Infants Health, Mount Sinai Hospital, Toronto, Ontario, Canada and ${ }^{4}$ Royal \\ Brisbane and Women's Hospital Campus, University of Queensland Centre for Clinical Research, Herston, \\ Queensland, Australia
}

Correspondence should be addressed to H M Georgiou at Department of Obstetrics and Gynaecology, Mercy Hospital for Women, 163 Studley Road, Heidelberg, Victoria, Australia 3084; Email: harrymg@unimelb.edu.au

\begin{abstract}
A significant obstetric complication facing contemporary materno-fetal medicine is preterm premature rupture of the fetal membranes (preterm PROM), which occurs in $30 \%$ of all preterm births. The objective of this study was to identify differentially expressed proteins in the cervicovaginal fluid of asymptomatic women before the clinical manifestation of preterm PROM. The preterm PROM group comprised of women with samples collected 6-23 days before PROM, who subsequently delivered preterm $(n=5)$. Women who spontaneously delivered at term served as gestation-matched controls $(n=10)$. Two-dimensional difference in-gel electrophoresis was used to distinguish differential expression between the pooled groups and fold changes were subsequently confirmed by two-dimensional PAGE of individual samples. Spots of interest were identified by mass spectrometry. Proteins that were significantly reduced with impending preterm PROM included the following: thioredoxin (2.7-fold), interleukin 1 receptor antagonist (1.7-fold), fatty acid-binding protein 5 (2.1-fold), cystatin A (dimer; 1.9-fold), monocyte/neutrophil elastase inhibitor (1.6-fold), squamous cell carcinoma antigen-1 (2.1-fold) and $\gamma$-glutamyl cyclotransferase (3.0-fold). By contrast, annexin A3 (3.7-fold) and vitamin D binding protein (3.9-fold) were significantly increased with impending preterm PROM. Western blot analysis was also performed on an independent cohort of preterm PROM and control samples to validate these candidate biomarkers. These proteins have known biological functions in oxidative balance, anti-inflammatory activity, metabolism or protease inhibition that may facilitate membrane rupture.

Reproduction (2013) 145 137-147
\end{abstract}

\section{Introduction}

Preterm premature rupture of membranes (PROM) is the rupture of the fetal membranes before the onset of labour at $<37$ weeks' gestation. Preterm PROM accounts for $\sim 30 \%$ of preterm births. It is widely acknowledged that preterm delivery (PTD) is associated with major neonatal morbidity and mortality with a significant burden placed upon the healthcare systems. Even 'late' preterm infants delivered between 34 and 37 weeks' gestation are at increased risk of adverse outcomes such as respiratory distress, jaundice, seizures and feeding problems compared with infants delivered at term (Bastek et al. 2008).

Risk factors of preterm PROM include ethnicity, low socioeconomic status, cigarette smoking, previous PTD, infection of the genital tract, cervical cerclage, amniocentesis, vaginal bleeding and uterine distension (i.e. polyhydramnios or multifetal gestation; Savitz et al. 1991, Mercer 2003). Each risk factor may contribute to the localised inflammation, infection and/or weakening of the fetal membranes leading to subsequent preterm PROM.

To date, no single biomarker has been characterised with high sensitivity and specificity for the prediction of women with subsequent preterm PROM and/or preterm labour. Currently, there are a number of diagnostic tests for women presenting with presumed premature rupture of the fetal membranes, with a clinical focus on the treatment of these women once membrane rupture has been confirmed (Simhan \& Canavan 2005, Zegels et al. 2010). However, it is our inability to predict preterm PROM that remains a significant obstetric problem. Exploration and further understanding of the biochemical pathways of human labour may provide the foundation for the discovery of such a biomarker. Ultimately, early therapeutic intervention and management before the clinical manifestation of preterm PROM may improve both neonatal and maternal outcome. 
The cervicovaginal fluid (CVF) is an accessible biological fluid that has been extensively studied for insights into term and preterm labour and biomarker discovery. The CVF proteome has been comprehensively characterised in pregnant and non-pregnant women (Dasari et al. 2007, Di Quinzio et al. 2007, 2008, Pereira et al. 2007, Shaw et al. 2007, Tang et al. 2007, Klein et al. 2008, Shah et al. 2009, Zegels et al. 2009, Heng et al. 2010a). Using two-dimensional PAGE (2D-PAGE) proteomic analysis of human CVF, we have demonstrated temporal protein changes in association with impending spontaneous term labour (Di Quinzio et al. 2008, Heng et al. 2010a). We hypothesise that these findings may reflect biochemical changes occurring in the cervix and overlying fetal membranes. Utilising a similar approach, the study of CVF may provide insight into the biochemical pathways associated with preterm PROM. Therefore, the aim of this discovery-based study was to characterise differential protein expression in human CVF in association with preterm PROM using 2D difference in-gel electrophoresis (2D-DIGE) coupled with liquid chromatography-electrospray ionisation with tandem mass spectrometry (nLC-ESI-MS/MS). Proteins of interest were further investigated using 2D-PAGE to confirm fold changes between individual women. Western blot analysis was used to validate the expression of selected proteins using an independent cohort of preterm PROM and gestation-matched control samples.

\section{Results}

\section{Clinical details and microbiology assessment}

Clinical details of the participants are displayed in Table 1. Microbiology assessment revealed common urogenital microflora in all women. No subject had bacterial vaginosis or clinical signs of chorioamnionitis at the time of CVF sampling.

\section{D-DIGE analysis of pooled CVF protein profiles}

Each CVF sample with a preterm PROM outcome was gestation matched with two control samples from women that subsequently resulted in a term delivery. The pooled CVF proteome map of asymptomatic pregnant women 6-23 days before preterm PROM and gestationmatched controls using 2D-DIGE is shown in Fig. 1. A total of 275 spots were matched between the two pooled groups. Seventy-one spots were up-regulated and 83 spots were down-regulated by a factor of 1.3-fold or more with impending preterm PROM.

\section{Protein characterisation}

Only 21 spots were visible to the naked eye and were manually excised and successfully characterised by nLC-ESI-MS/MS (peptide sequences are found in Supplementary Table 1 , see section on supplementary data given at the end of this article). These spots represented 14 unique proteins, some of which have multiple isoforms differing in isoelectric point (pl) and/or molecular weight $(\mathrm{MW})$. Annexin $\mathrm{A} 3$, apolipoprotein A1, three isoforms of albumin and two isoforms of vitamin D binding protein (VDBP) were up-regulated 6-23 days before spontaneous preterm PROM compared with controls (Table 2). Spots that were down-regulated with impending preterm PROM were $\mathrm{Cu}, \mathrm{Zn}$-superoxide dismutase (Cu,Zn-SOD), cystatin A (also known as stefin A), $\gamma$-glutamyl cyclotransferase (GGCT), interleukin 1 receptor antagonist (IL1ra), two isoforms of monocyte/ neutrophil elastase inhibitor ( $\mathrm{M} / \mathrm{NEl}$; also known as Serpin B1), placental thrombin inhibitor (also known as Serpin B6), squamous cell carcinoma antigen-1 (SCCA-1; also known as Serpin B3) and thioredoxin. Fold changes for each identified protein are shown in Table 2.

Isoforms of two characterised proteins showed opposing expression trends: calgranulin B (spot 513) was down-regulated 1.4-fold compared with another isoform

Table 1 Clinical details of participants.

\begin{tabular}{|c|c|c|c|c|c|c|}
\hline Subject & $\begin{array}{c}\text { Maternal age } \\
\text { (years) }\end{array}$ & $\begin{array}{l}\text { Gestation at CVF } \\
\text { sampling }\end{array}$ & $\begin{array}{l}\text { Days from } \\
\text { preterm PROM }\end{array}$ & $\begin{array}{l}\text { Gestation at } \\
\text { preterm PROM }\end{array}$ & $\begin{array}{l}\text { Gestation } \\
\text { at delivery }\end{array}$ & Outcome \\
\hline $1^{\mathrm{a}}$ & 29.67 & 23 weeks 6 days & 13 & 26 weeks 0 day & 30 weeks 0 day & Preterm PROM \\
\hline $2^{\mathrm{a}}$ & 27.83 & 31 weeks 5 days & 8 & 33 weeks 0 day & 33 weeks 3 days & Preterm PROM \\
\hline 3 & 34.75 & 32 weeks 2 days & 6 & 33 weeks 1 day & 33 weeks 5 days & Preterm PROM \\
\hline 4 & 28.42 & 34 weeks 0 day & 15 & 36 weeks 1 day & 36 weeks 2 days & Preterm PROM \\
\hline 5 & 25.42 & 31 weeks 6 days & 23 & 35 weeks 1 day & 35 weeks 2 days & Preterm PROM \\
\hline $6^{\mathrm{a}}$ & 26.75 & 23 weeks 6 days & - & - & 40 weeks 0 day & Term \\
\hline $7^{\mathrm{a}}$ & 20.92 & 24 weeks 1 day & - & - & 37 weeks 2 days & Term \\
\hline $8^{a}$ & 27.29 & 31 weeks 5 days & - & - & 39 weeks 4 days & Term \\
\hline $9^{a}$ & 32.08 & 31 weeks 5 days & - & - & 37 weeks 3 days & Term \\
\hline 10 & 42.25 & 32 weeks 2 days & - & - & 39 weeks 0 day & Term \\
\hline 11 & 35.75 & 32 weeks 2 days & - & - & 39 weeks 3 days & Term \\
\hline 12 & 26.75 & 34 weeks 2 days & - & - & 39 weeks 6 days & Term \\
\hline 13 & 20.92 & 33 weeks 3 days & - & - & 40 weeks 6 days & Term \\
\hline 14 & 31.83 & 32 weeks 1 day & - & - & 40 weeks 4 days & Term \\
\hline 15 & 34.67 & 31 weeks 5 days & - & - & 39 weeks 4 days & Term \\
\hline
\end{tabular}

${ }^{\mathrm{a}}$ Twin gestation. 
A

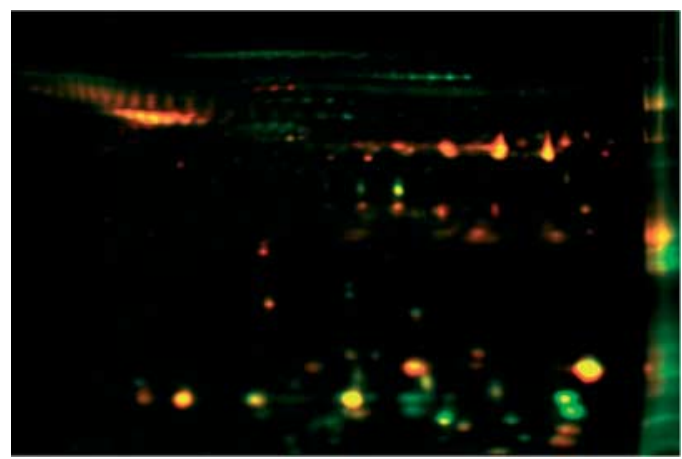

B

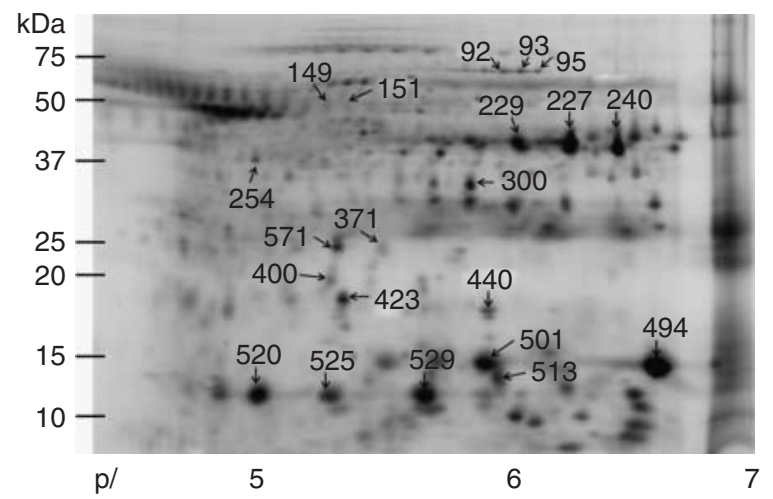

Figure 1 (A) 2D-DIGE pseudo-colour overlay of CVF samples collected from women 6-23 days before preterm PROM (green) and gestationmatched controls (red). Yellow spots represent equal protein expression between the two groups. (B) Characterised spots are labelled with corresponding spot number assigned by SameSpots 2D-DIGE analysis software.

(spot 529) that was up-regulated 2.4-fold in the preterm PROM group. Fatty acid-binding protein 5 (FABP5; spot 494) was decreased 2.0-fold in the preterm PROM group, whereas the other isoform of FABP5 (spot 501; identical MW differing only in $\mathrm{pl}$ ) did not exhibit expressional change before preterm PROM (Table 2).

\section{Confirmation of fold changes using 2D-PAGE}

To confirm the fold changes between the two pooled clinical groups, each sample was individually subjected to 2D-PAGE analysis (Fig. 2). Spot detection ranged from 157 to 261 spots present between all gels. A single master match-set was created from the 15 gels and comprised of 140 spots. Each spot was assigned an arbitrary number generated by the PD Quest Software for statistical analysis and spot characterisation. Spots that showed differential protein abundance (based on spot volume) and were confidently identified by mass spectrometry were matched in all gels and subjected to non-parametric Mann-Whitney $U$ statistical analysis.

A total of nine identified spots showed significant changes in the mean spot volumes $( \pm$ s.D.) as preterm PROM approached (Table 3). Seven spots were significantly decreased (ranging from -1.6 to -3.0 -fold)
6-23 days before preterm PROM when compared with the gestation-matched control samples: M/NEI (spot 227, $P=0.028)$, SCCA-1 $(P=0.028)$, GGCT $(P=0.013)$, IL1 ra $(P=0.005)$, FABP5 $(P=0.005)$, thioredoxin $(P=0.013)$ and the dimeric form of cystatin $A(P=0.028)$. By contrast, spot volumes for two proteins were significantly increased (ranging from 3.7- to 3.9-fold) with impending preterm PROM: VDBP (spot 149, $P=0.005$ ) and annexin A3 $(P=0.005)$. Comparison of the fold changes calculated from the pooled groups (2D-DIGE) and individual samples (2D-PAGE) were overall consistent between the two proteomic techniques (Table 3).

\section{Immunovalidation}

An independent cohort of an additional 15 women was recruited to validate the presence of six candidate biomarkers of interest. Women that subsequently experienced preterm PROM within 2-20 days of sample collection $(n=5$; two singletons and three twins) were gestation matched with healthy women who spontaneously delivered at term $(n=10$; four singletons and six twins). Western blot analysis was performed using commercially available antibodies raised against VDBP, annexin A3, IL1 ra, cystatin A, FABP5 and thioredoxin. The optical density of individual protein bands was normalised against the total optical density of each lane (Fig. 3). The expression of annexin A3 $(P=0.04)$, IL1 ra $(P=0.001)$ and cystatin $\mathrm{A}(P=0.001)$ was significantly different (Mann-Whitney $U$ test) in the independent cohort of preterm PROM women. Two candidate biomarkers, VDBP and FABP5, displayed similar expressional trends as observed in the 2D-electrophoresis experiments but failed to reach statistical significance.

\section{Discussion}

Preterm PROM is a significant problem facing contemporary clinical obstetrics worldwide. The majority of cases of preterm PROM are associated with underlying pathological processes, such as infection and/or inflammation of the fetal membranes. In the absence of infection, delivery of the neonate is often delayed if preterm PROM occurs at an early gestation $(<34$ weeks') in order to bide time for the developing fetus. This can conversely lead to increased risk of chorioamnionitis and serious sequelae for the neonate.

There has been limited research of biomarkers that may predict preterm PROM. Biggio et al. (2005) reported that women with elevated midtrimester amniotic fluid matrix metalloproteinase 8 (MMP-8) levels greater than the 90th percentile were strongly associated with subsequent preterm PROM outcomes compared with term controls. Rosen et al. (2001) also reported that 
Table 2 Characterisation of identified proteins with fold changes $\geq 1.3$ using 2D-DIGE.

\begin{tabular}{|c|c|c|c|c|c|c|c|c|}
\hline Spot no. ${ }^{a}$ & Protein identity & $\begin{array}{l}\text { Matched } \\
\text { peptides }\end{array}$ & $\begin{array}{c}\text { Coverage } \\
(\%)\end{array}$ & $\begin{array}{l}\text { Accession } \\
\text { no. }{ }^{b}\end{array}$ & $\begin{array}{c}\text { Experimental } \\
\text { molecular } \\
\text { weight } \\
(\mathrm{kDa}) / \mathrm{pl}\end{array}$ & $\begin{array}{c}\text { Theoretical } \\
\text { molecular } \\
\text { weight } \\
(\mathrm{kDa}) / \mathrm{pl}\end{array}$ & $\begin{array}{l}\text { MASCOT } \\
\text { score }\end{array}$ & $\begin{array}{c}\text { Fold } \\
\text { change }^{\mathrm{C}} \\
\end{array}$ \\
\hline 92 & Albumin & 3 & 7 & P02768 & $61.7 / 5.9$ & $71.3 / 5.9$ & 159 & 13 \\
\hline 93 & Albumin & 8 & 20 & P02768 & $61.7 / 6.0$ & $71.3 / 5.9$ & 426 & 14 \\
\hline 95 & Albumin & 2 & 20 & P02768 & $61.7 / 6.1$ & $71.3 / 5.9$ & 107 & 8.8 \\
\hline 300 & Annexin A3 & 7 & 30 & P12429 & $37.2 / 6.0$ & $36.5 / 5.6$ & 353 & 3.8 \\
\hline 371 & Apolipoprotein A1 & 8 & 33 & P02647 & $26.3 / 5.4$ & $30.8 / 5.6$ & 483 & 1.6 \\
\hline 513 & Calgranulin B & 2 & 24 & P06702 & $14.8 / 5.9$ & $13.3 / 5.7$ & 66 & 2.4 \\
\hline 529 & Calgranulin B & 4 & 43 & P06702 & $13.5 / 6.3$ & $13.3 / 5.7$ & 106 & -1.4 \\
\hline 440 & $\mathrm{Cu}, \mathrm{Zn}-\mathrm{SOD}$ & 3 & 23 & P00441 & $18.6 / 5.9$ & $16.2 / 5.7$ & 201 & -1.8 \\
\hline 525 & Cystatin A (monomer) & 3 & 53 & P01040 & $12.4 / 5.3$ & $11.0 / 5.38$ & 71 & -1.5 \\
\hline 571 & Cystatin A (dimer) & 3 & 31 & P01040 & $23.2 / 5.4$ & $11.0 / 5.38$ & 125 & -1.8 \\
\hline 494 & $\mathrm{FAC}^{\prime} \mathrm{BP} 5^{\mathrm{d}}$ & 2 & 26 & Q01469 & $15.5 / 6.6$ & $15.5 / 6.6$ & 150 & -2.0 \\
\hline 400 & GGCT & 6 & 38 & O75223 & $21.4 / 5.2$ & $21.2 / 5.1$ & 173 & -2.8 \\
\hline 423 & IL1 ra & 4 & 33 & P18510 & $20.0 / 5.3$ & $20.4 / 5.8$ & 221 & -1.6 \\
\hline 227 & M/NEI & 6 & 21 & P30740 & $43.7 / 6.3$ & $42.8 / 5.9$ & 357 & -1.5 \\
\hline 229 & $\mathrm{M} / \mathrm{NEI}$ & 7 & 21 & P30740 & $42.8 / 6.0$ & $42.8 / 5.9$ & 470 & -1.4 \\
\hline 254 & $\begin{array}{l}\text { Placental thrombin } \\
\text { inhibitor (Serpin B6) }\end{array}$ & 3 & 11 & P35237 & $43.7 / 4.8$ & $43.0 / 5.2$ & 108 & -2.3 \\
\hline 240 & SCCA-1 & 7 & 27 & Q8IXI3 & $38.9 / 6.5$ & $44.6 / 6.4$ & 290 & -1.3 \\
\hline 520 & Thioredoxin & 3 & 22 & P10599 & $13.0 / 4.9$ & $12.0 / 4.8$ & 265 & -2.3 \\
\hline 149 & VDBP & 7 & 22 & P02774 & $51.3 / 5.2$ & $54.5 / 5.4$ & 367 & 2.3 \\
\hline 151 & VDBP & 8 & 31 & P02774 & $51.3 / 5.3$ & $54.5 / 5.4$ & 423 & 1.4 \\
\hline
\end{tabular}

$\mathrm{Cu}, \mathrm{Zn}-\mathrm{SOD}, \mathrm{Cu}, \mathrm{Zn}$-superoxide dismutase; FABP5, fatty acid-binding protein 5; GGCT, $\gamma$-glutamyl cyclotransferase; IL1 ra, interleukin 1 receptor antagonist; $\mathrm{M} / \mathrm{NEI}$, monocyte/neutrophil elastase inhibitor; SCCA-1, squamous cell carcinoma antigen-1; VDBP, vitamin D binding protein.

${ }^{\mathrm{a}}$ Spot number generated by Progenesis SameSpots Software v3.3. ${ }^{\mathrm{b}}$ Accession number of Swiss-Prot database. ${ }^{\mathrm{C}}$ Fold change with respect to controls.

${ }^{\mathrm{d}}$ Another isoform of fatty acid-binding protein 5 (spot 501) was identified; however, it was not found to be differentially expressed.

increased concentrations of plasma thrombin-antithrombin complexes in the maternal blood correlate with subsequent preterm PROM. Another study conducted by Longini et al. (2007) found isoprostane (a by-product of lipid peroxidation) to be significantly higher in the amniotic fluid collected at 15-18 weeks' gestation from women who deliver preterm following subsequent preterm PROM compared with women with normal term labour. In this current study, we investigated alterations in the CVF protein expression of asymptomatic women, without clinical signs of infection, who progressed to preterm PROM and subsequently delivered preterm. To our knowledge, this is the first study characterising protein changes in the CVF of asymptomatic women who subsequently experienced preterm PROM. The differentially expressed proteins identified in this study relate to presumed biochemical processes that predate the rupture of the fetal membranes.

We analysed human CVF using 2D-DIGE coupled with $\mathrm{nLC}-\mathrm{ESI}-\mathrm{MS} / \mathrm{MS}$ and identified 14 different proteins with a $\geq 1.3$-fold change at $6-23$ days before spontaneous preterm PROM. We subsequently performed 2D-PAGE, and of the 14 proteins identified by 2D-DIGE, nine proteins were found to be significantly differentially expressed. The proteins identified have biological roles that include inflammation (IL1 ra, VDBPand annexin A3), proteinase inhibition (M/NEI, SCCA-1 and cystatin A) and oxidative defence (thioredoxin, GGCT and FABP5). All these proteins have been previously identified to exhibit significant changes in the CVF associated with term (Di Quinzio et al. 2008, Heng et al. 2010a) and preterm labour (Pereira et al. 2007). Their putative biological roles in the pathophysiological process of preterm PROM will be discussed.

IL1ra is a member of the IL1 superfamily and is expressed by immune cells (Malyak et al. 1994) and in the decidua, chorion and amnion (Ämmälä et al. 1997). This protein has an inhibitory role on the downstream signalling effects of pro-inflammatory IL $1 \alpha$ and IL1 $\beta$ cytokines through the competitive binding of the membrane-bound IL1 receptors of target cells. IL1 $\beta$ in conjunction with tumour necrosis factor (TNF) induces an increase in MMPs (MMP-1, -3 and -9) and cathepsin S expression and activity in the cervical smooth muscles contributing to extracellular matrix (ECM) remodelling (Watari et al. 1999). IL1 $\beta$ and TNF have also been linked to induce apoptosis of trophoblastic cells (Fortunato et al. 1999). It is feasible that the action of IL1 ra may be to naturally inhibit these dynamic physiological processes during pregnancy.

Studies have demonstrated that at term, the supracervical site of the fetal membranes (overlying the cervix) has reduced tensile strength and altered morphology of the connective tissues that is consistent with collagen degradation and increased cellular apoptosis (McLaren et al. 1999, Moore et al. 2006, Reti et al. 2007). In this study, IL1ra expression in the CVF was significantly reduced in women 6-23 days before preterm PROM when compared with gestation-matched controls. This reduction of IL1ra may augment the downstream 

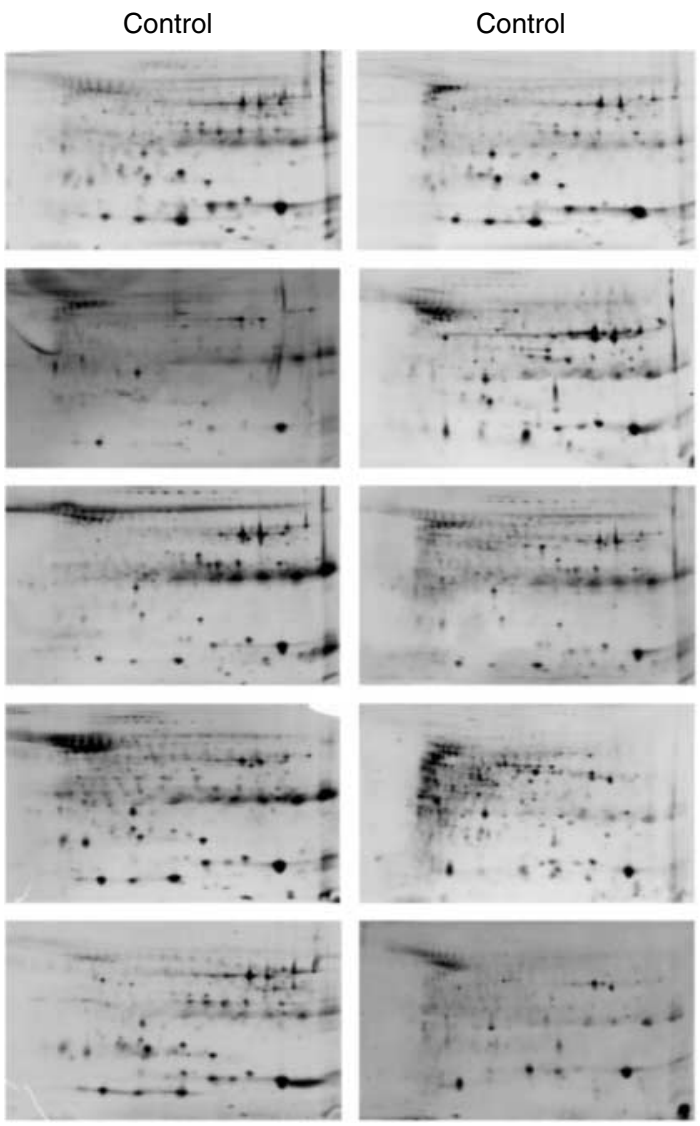

\section{Preterm PROM}
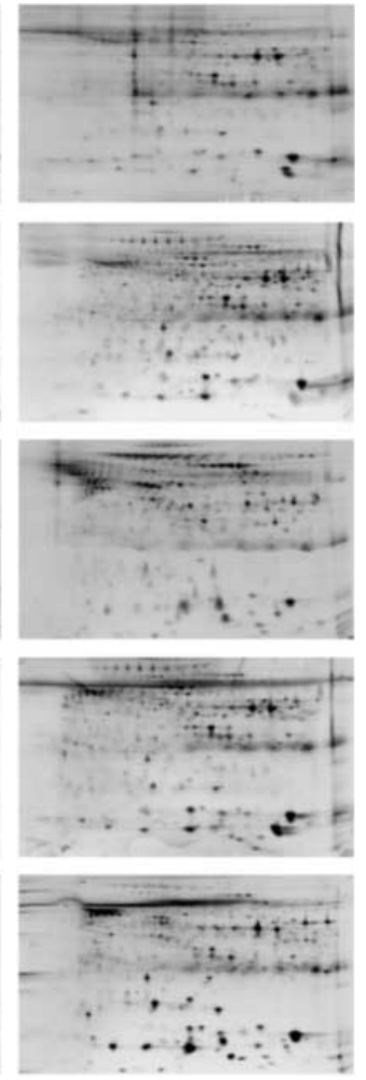

Figure 2 2D-PAGE gels (raw images) of CVF samples collected from women who experienced preterm PROM within 6-23 days of sample collection and their appropriate gestation-matched controls. pro-inflammatory effects of IL1 $\beta$, which may manifest as a weakening of the fetal membranes and their early rupture.

VDBP, also known as Gc-globulin (group-specific component), is a $51-58 \mathrm{kDa}$ serum glycoprotein. It acts as a carrier protein for vitamin $\mathrm{D}$ sterols and regulates the uptake of free vitamin D metabolites into target cells. However, only $5 \%$ of the total circulating VDBP is bound to vitamin $\mathrm{D}$, suggesting a reservoir function for these sterols (White \& Cooke 2000). VDBP also displays a high binding affinity for free monomeric actin, which is released during tissue damage or cell death (McLeod et al. 1989). Furthermore, VDBP has been shown to sequester free circulating actin monomers in rats, released during tissue damage and cell death (Harper et al. 1987). During infection or tissue injury, VDBP becomes deglycosylated as a consequence of the immune response, transforming its function to a macrophage activating factor (Gc-MAF; Yamamoto \& Homma 1991, Yamamoto \& Kumashiro 1993). VDBP also enhances the chemotatic activity of the complement factor $\mathrm{C} 5 \mathrm{a}$ for the recruitment of immunocompetent cells at the site of inflammation (Piquette et al. 1994). The observed increase in VDBP expression in the CVF may reflect the increased cell death and/or inflammation occurring at the supracervical site of the fetal membranes, as preterm PROM approaches. A limitation of the current study is that serum vitamin D status was not determined in the two study cohorts. At our hospital in Melbourne, Australia, a mild degree of vitamin D

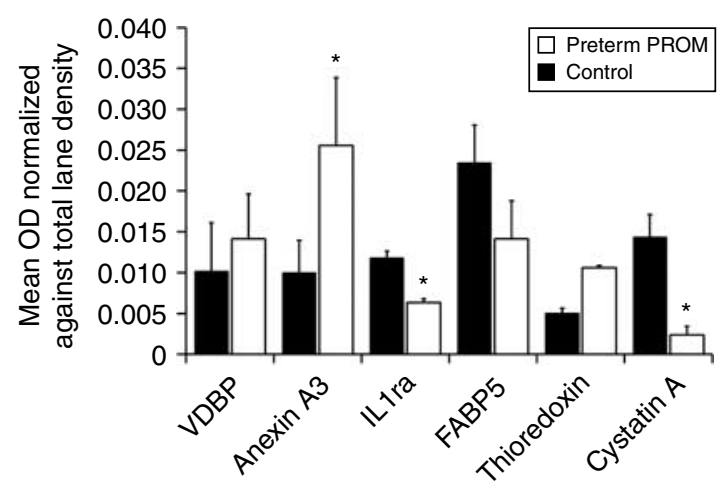

Figure 3 Validation of potential CVF biomarkers of approaching preterm PROM. Using an independent cohort of women (preterm $\mathrm{PROM}=5$, gestation-matched controls $=10$ ), $25 \mu \mathrm{g}$ CVF protein from each subject were collected, electrophoresed and probed by western blot using commercially available antibodies raised against VDBP, annexin A3, IL1 ra, cystatin A, FABP5 and thioredoxin. The optical density of individual protein bands was normalised against the total optical density of each lane. ${ }^{*}$ indicates statistical significance between control vs preterm PROM group $(P<0.05)$. 
Table $32 \mathrm{DE}$ analysis of differentially expressed spots.

\begin{tabular}{|c|c|c|c|c|c|c|}
\hline \multirow[b]{2}{*}{ Spot no. $^{\text {a }}$} & \multirow[b]{2}{*}{ Protein identity } & \multicolumn{2}{|c|}{ Average spot volume \pm s.D. } & \multirow{2}{*}{$\begin{array}{c}\text { 2D-PAGE, } \\
P \text { value }\end{array}$} & \multirow{2}{*}{$\begin{array}{l}\text { 2D-PAGE } \\
\text { fold change }\end{array}$} & \multirow{2}{*}{$\begin{array}{l}\text { 2D-DIGE } \\
\text { fold chang }\end{array}$} \\
\hline & & Control $(n=10)$ & Preterm PROM $(n=5)$ & & & \\
\hline 300 & Annexin A3 & $3464 \pm 1931$ & $12868 \pm 5306$ & 0.005 & 3.7 & 3.8 \\
\hline 371 & Apolipoprotein A1 & $705 \pm 358$ & $1014 \pm 592$ & 0.513 & 1.4 & 1.6 \\
\hline 513 & Calgranulin B & $14202 \pm 7813$ & $27866 \pm 19603$ & 0.165 & 2.0 & 2.4 \\
\hline 529 & Calgranulin B & $28585 \pm 24252$ & $21109 \pm 28275$ & 0.371 & -1.4 & -1.4 \\
\hline 440 & $\mathrm{Cu}, \mathrm{Zn}-\mathrm{SOD}$ & $9312 \pm 4903$ & $6130 \pm 367$ & 0.679 & -1.5 & -1.8 \\
\hline 525 & Cystatin A (monomer) & $9527 \pm 10663$ & $7518 \pm 6788$ & 0.953 & -1.3 & -1.5 \\
\hline 571 & Cystatin A (dimer) & $11331 \pm 4578$ & $6010 \pm 1594$ & 0.028 & -1.9 & -1.8 \\
\hline 494 & FABP5 & $130365 \pm 57287$ & $62079 \pm 29275$ & 0.005 & -2.1 & -2.0 \\
\hline 400 & GGCT & $2823 \pm 1256$ & $932 \pm 811$ & 0.013 & -3.0 & -2.8 \\
\hline 423 & IL1ra & $18687 \pm 1114$ & $11247 \pm 3010$ & 0.005 & -1.7 & -1.6 \\
\hline 227 & M/NEI & $47630 \pm 27949$ & $29159 \pm 7474$ & 0.028 & -1.6 & -1.5 \\
\hline 229 & M/NEI & $6192 \pm 2115$ & $4423 \pm 3424$ & 0.513 & -1.4 & -1.4 \\
\hline 254 & Serpin B6 & $3029 \pm 1933$ & $1530 \pm 308$ & 0.165 & -2.0 & -2.3 \\
\hline 240 & SCCA-1 & $30656 \pm 16733$ & $14263 \pm 7633$ & 0.028 & -2.1 & -1.3 \\
\hline 520 & Thioredoxin & $19088 \pm 10367$ & $7007 \pm 5925$ & 0.013 & -2.7 & -2.3 \\
\hline 149 & VDBP & $439 \pm 239$ & $1718 \pm 1250$ & 0.005 & 3.9 & 2.3 \\
\hline 151 & VDBP & $916 \pm 530$ & $1804 \pm 1763$ & 0.371 & 2.0 & 1.4 \\
\hline
\end{tabular}

Spots showing a significant difference are shown in bold. $P$ value was calculated using the non-parametric Mann-Whitney $U$ test.

${ }^{a}$ Spot number according to number assigned by Progenesis SameSpots v3.3 Software.

insufficiency $(20-60 \mathrm{nmol} / \mathrm{l})$ is not uncommon in pregnancy. This may have impacted on the VDBP expression, to varying degrees, in the CVF of the two cohorts investigated.

Annexin A3 is a $33 \mathrm{kDa}$ protein expressed in the trophoblasts of placental villous tissue and secreted as granules derived from activated neutrophils and monocytes (Le Cabec \& Maridonneau-Parini 1994). Annexin A3 has anticoagulant properties and inhibitory effects on the activity of the phospholipase A2, which is involved in the hydrolysis of cell membrane-derived arachidonic acid for prostaglandin synthesis (Tait et al. 1988). The significant increase in annexin A3 demonstrated in our study may be a response to the increased inflammation occurring at the site of fetal membrane rupture. This is in agreement with the reported increased expression of annexin A3 associated with threatened preterm labour and preterm birth in human CVF (Pereira et al. 2007).

M/NEI and SCCA- 1 are members of the Serpin (serine proteinase inhibitors) superfamily. $\mathrm{M} / \mathrm{NEI}$ is a naturally occurring inhibitor of neutrophil-secreted granule proteases (namely cathepsin G, proteinase 3 and neutrophil elastase; Sugimori et al. 1995), thereby regulating connective tissue remodelling at sites of inflammation. This current study is consistent with the findings by Helmig et al. (2002) where a significant reduction in $\mathrm{M} / \mathrm{NEI}$ concentration in the amniotic fluid of asymptomatic women with preterm PROM, in the absence of chorioamnionitis, was observed. SCCA- 1 is a broad inhibitor of cysteine proteinases, cathepsins $\mathrm{L}, \mathrm{S}$ and $\mathrm{K}$, which are implicated in ECM remodelling through elastin degradation (Schick et al. 1998) and is localised in the squamous epithelial cells of the cervix and vagina (Crombach et al. 1989, Cataltepe et al. 2000). A decrease in SCCA-1 expression in the CVF of women with impending preterm PROM may reflect a concomitant increase in cathepsin activity occurring in the ECM of the supracervical membranes leading to their weakening and subsequent rupture.

The decreased expression of M/NEI (Serpin B1) and SCCA-1 (Serpin B3) in the maternal CVF is intriguing and may indeed be surrogate markers of fetal membrane remodelling. In the fetus (including the fetal membranes), the SERPINH1 gene encodes for heat-shock protein 47 (Hsp47) that normally serves as a chaperone to stabilise the collagen triple helix. Findings by Wang et al. (2006) describe a single nucleotide polymorphism (SNP) in the promoter region of the SERPINH1 gene that is strongly associated with the risk of preterm PROM and is highly prevalent in African Americans. By contrast, a 12 bp deletion in SERPINH1 promoter region increases promoter activity and mitigates the impact of the SNP of Hsp47 (Wang et al. 2008). Further exploration of the role of SERPIN genes from both the mother and the fetus in preterm PROM is of future interest.

Cystatin A is expressed by leucocytes and epithelial cells, including the squamous epithelial cells of the cervix and vagina (Davies \& Barrett 1984). Cystatin A is expressed in the CVF of pregnant women in both monomeric and dimeric forms (Di Quinzio et al. 2007) and is significantly decreased in association with spontaneous term labour (Heng et al. 2011). It is a non-specific cysteine proteinase inhibitor regulating the proteolytic activities of cathepsins B, H and L (Turk \& Bode 1991). At sites of inflammation, cathepsins $B, L$ and $S$ are released during cell death and secreted by activated macrophages, causing the degradation of collagen and elastin, which may lead to ECM remodelling (Reddy et al. 1995). Dimerisation of cystatin $\mathrm{A}$ that occurs under denaturing conditions (high temperatures $74-93{ }^{\circ} \mathrm{C}$ and low $\mathrm{pH}<2.5$; Martin et al. 
1995, Japelj et al. 2004) has been found to inactivate its inhibitory functions. We found a reduced expression of cystatin A 6-23 days before preterm PROM, which may possibly reflect a decrease in fetal membrane integrity due to an increase in unregulated proteolytic activity by cysteine proteinases.

Three antioxidant enzymes, namely thioredoxin, GGCT and FABP5, were decreased in association with preterm PROM. Thioredoxin is a $12 \mathrm{kDa}$ antioxidant enzyme expressed in the cervix (Lysell et al. 2003) and fetal membranes (Perkins et al. 1995). Thioredoxin has been associated with a number of critical processes including defence against tissue damage by reactive oxygen species (ROS) and inhibition of apoptosis (Nakamura et al. 1997, Saitoh et al. 1998). Redox regulation of the transcription factor nuclear factor $\kappa B$ $(\mathrm{NF}-\mathrm{\kappa B})$ activity is mediated by the balance between ROS and antioxidant defence, including thioredoxin. NF- $\kappa \mathrm{B}$ affects the expression of cyclooxygenase-2, prostaglandins and various pro-inflammatory cytokines. GGCT is an enzyme involved in the $\gamma$-glutamyl cycle for glutathione homeostasis by converting $\gamma$-glutamyl dipeptides to 5-oxoproline (Pastore et al. 2003). Glutathione constitutes the major endogenous antioxidant produced by cells for the defence against ROS. The marked decrease in GGCT may indirectly lead to a concomitant decrease in glutathione concentration.

Only one of the two isoforms of FABP5 was significantly reduced in human CVF 6-23 days before preterm PROM. Both isoforms of FABP5 identified in this study displayed an identical MW, however, differed in their pl. This may explain why statistical significance was not observed in the validation cohort. FABP5 has been identified in the CVF by at least eight other groups but the existence of different isoforms could not be described using the technologies employed (Venkataraman et al. 2005, Dasari et al. 2007, Pereira et al. 2007, Shaw et al. 2007, Tang et al. 2007, Klein et al. 2008, Shah et al. 2009, Zegels et al. 2009). The significance of the different isoforms therefore remains unclear. FABP5 is highly expressed in epidermal cells, including the cervix and vagina (Siegenthaler et al. 1993). FABP5 is thought to have an antioxidative role by acting as a scavenger of chemically reactive lipids (Bennaars-Eiden et al. 2002), amongst other biological functions. FABP5 is reduced in the CVF in association with both spontaneous term and PTD (Pereira et al. 2007, Di Quinzio et al. 2008). However, the exact physiological role of FABP5 in the process of membrane rupture and labour is unknown. It is hypothesised that the significant decrease in thioredoxin, GGCT and one isoform of FABP5 expression in the CVF with impending preterm PROM demonstrated in this study may reflect a reduction in the total antioxidant defence against ROS, a phenomenon we have previously described (Heng et al. 2010b). This dysregulated balance of ROS may increase the oxidative stress-mediated effects including inflammation, tissue damage, prostaglandin production and proteinase activity that could ultimately lead to ECM degradation and premature fetal membrane rupture.

Thus far, only proteins that were verified with 2D-PAGE have been discussed. However, it is interesting that in our initial 2D-DIGE experiment, albumin displayed the greatest fold change. Unfortunately, we were unable to confirm this finding by 2D-PAGE because the albumin spots could not be adequately resolved and/or matched given their relatively high MW using an $8-16 \%$ gradient gel. This highlights a limitation of $2 \mathrm{D}$ PAGE. We have previously described increased albumin expression in the CVF in association with approaching labour by utilising a 10\% gel (Heng et al. 2010a) and speculate that this may reflect increased inflammation and leucocyte migration occurring in the supracervical membranes. Further investigation of albumin expression in the CVF in association with preterm PROM is required.

In conclusion, we have investigated the global dynamic changes in the CVF proteome of asymptomatic women who subsequently experienced preterm PROM within 6-23 days. This study has revealed a number of significant differentially expressed proteins that are involved in inflammation, ECM remodelling and oxidative balance. Collectively, these protein changes and their consequent effects may represent biochemical evidence of a distinct pathway that leads to rupture of the fetal membranes in the absence of cervical ripening and myometrial activation.

Owing to the small sample size (that included both singleton and twin gestations and a relatively wide interval of sampling to preterm PROM), the findings presented in this study should be regarded as preliminary. We acknowledge that the mechanism(s) underlying the rupture of fetal membranes may differ between singleton and multifetal gestations. Similarly, the cause(s) of early $(<28$ weeks) and late (35-36 weeks) preterm PROM may differ. We have attempted to address these confounding factors by gestationally matching singleton and twin pregnancies (with a similar interval in CVF sampling to preterm PROM) in an independent cohort of women in the western blot validation study. This may explain why western blot validation could not confirm our results for three of the six candidate biomarkers investigated. Further investigation and validation in a larger prospective preterm PROM population using traditional immuno-based techniques is warranted. It is anticipated that these and other biomarkers could be utilised in the development of a sensitive and specific screening test for the detection of women at risk of delivering preterm.

\section{Materials and Methods}

\section{Subject recruitment and sample collection}

This study was approved by the Mercy Health, Research Ethics Committee (R56/06). All participants were prospectively 
recruited by a Research Midwife who obtained written informed consent. Samples were retrospectively selected based on birthing outcome for subsequent analysis. Pregnant women attending the Antenatal Clinic at the Mercy Hospital for Women (Heidelberg, Victoria, Australia) with identified risk factors for spontaneous preterm birth were recruited at $\sim 24$ weeks' gestation. CVF samples were collected every 4 weeks up to 36 weeks' gestation or collection ceased if PTD had occurred. Once delivery outcomes were verified, the CVF samples were stratified post hoc into two groups.

The preterm PROM group consisted of asymptomatic women who presented with spontaneous preterm PROM 6-23 days after CVF sample collection and subsequently delivered preterm $(n=5)$. Women from the same prospective cohort, who spontaneously delivered at term without preterm PROM, were selected to serve as gestation-matched controls $(n=10)$. Inclusion criteria for recruitment were any of the following: a history of spontaneous PTD or preterm PROM, shortened cervix, previous cone biopsy, uterine anomaly and heavy smoker. Exclusion criteria were women with bacterial vaginosis, clinical signs of chorioamnionitis, unprotected sexual intercourse in the preceding $48 \mathrm{~h}$ before CVF collection and vaginal examination or transvaginal ultrasound in the preceding $6 \mathrm{~h}$ before CVF sample collection.

CVF was collected from the posterior vaginal fornix using a double-tipped rayon swab (Duo-Transtube, Medical Wire and Equipment Co. Ltd., Corsham, Wilts, England) for $30 \mathrm{~s}$ and immersed in $1 \mathrm{ml}$ CVF extraction buffer $(50 \mathrm{mmol} / \mathrm{l}$ HEPES, $150 \mathrm{mmol} / \mathrm{l} \mathrm{NaCl}, 0.1 \% \mathrm{v} / \mathrm{v}$ SDS, $1 \mathrm{mmol} / \mathrm{I} \mathrm{EDTA}$ and $1 \mathrm{mmol} / \mathrm{l}$ AEBSF protease inhibitor). Samples were processed immediately according to a previously published protocol (Di Quinzio et al. 2007). A high vaginal swab was also collected from the posterior vaginal fornix for microbiological assessment. Routine microbiological culture and microscopy were performed adhering to standard laboratory methods (Microbiology Department, Austin Pathology, Austin Health, Heidelberg, Victoria, Australia).

\section{Sample processing and 2D-DIGE}

An equivalent protein load of individual samples was pooled into the two distinct groups. The pooled samples were precipitated using the 2D Clean-Up kit (GE Healthcare, Piscataway, NJ, USA) according to the manufacturer's instruction. Samples were resolubilised in rehydration buffer (7 M urea, $2 \mathrm{M}$ thiourea, 4\% w/v CHAPS and $30 \mathrm{mM}$ Tris). Buffer exchanges were performed to obtain conductivity measurements $<300 \mu \mathrm{S} / \mathrm{cm}$. Total protein concentration of pooled samples was determined using the Bradford Coomassie Plus Protein Assay Reagent Kit (Pierce Biotechnology, Rockford, IL, USA). For each pooled group, $25 \mu \mathrm{g}$ protein was labelled with 200 pmol CyDye DIGE Fluor minimal dye (GE Healthcare). To normalise protein abundance, an internal standard was created using equal aliquots labelled with Cy2 dye from the two study groups. Dye swapping for each group was used to account for labelling bias using both Cy 3 and Cy 5 dyes. The labelled samples were subsequently pooled according to the study design.
A total of $75 \mu \mathrm{g}$ protein was loaded onto $11 \mathrm{~cm}$ immobilised $\mathrm{pH}$ gradient strips with a linear gradient of $\mathrm{pH}$ 4-7 (IPG Ready Strip; Bio-Rad Laboratories, Hercules, CA, USA) and subjected to first-dimension separation at $34000 \mathrm{Vh}$. IPG strips were then equilibrated consecutively for $15 \mathrm{~min}$ with dithiothreitol and iodoacetamide (IAA) equilibration buffers. Second-dimension separation was performed using Criterion $8-16 \%$ Tris- $\mathrm{HCl}$ polyacrylamide gradient gels (Bio-Rad Laboratories). Gels were scanned at $100 \mathrm{~nm}$ resolution in a Typhoon 9400 scanner (GE Healthcare) using the following channels: 580 BP 30 (Cy3; PMT voltage: $490 \mathrm{~V}), 520$ BP 40 (Cy2; $480 \mathrm{~V}$ ) and 670 BP 30 (Cy5; 670 V). ImageQuant TL Software, version 7.0 (GE Healthcare), was used to assign pseudo-colours to different lasers and images were overlaid to visualise differences. Protein spot analysis was performed using Progenesis SameSpots 3.3 Software (Non-Linear Dynamics, Newcastle upon Tyne, UK) to identify differentially expressed spots. Normalised Cy3- and Cy5-labelled spots were compared to the internal standard to determine the proportional amount of protein.

\section{Mass spectrometry}

Gels were fixed in 10\% acetone/25\% isopropanol solution and counter stained with Coomassie (G-250, PageBlue; Fermentas, Burlington, ON, Canada). Protein spots of interest were manually excised and processed for mass spectrometry characterisation. Briefly, excised gel plugs were destained twice with $50 \%$ acetonitrile in $100 \mathrm{mM}$ triethylammonium bicarbonate (TEAB; Sigma-Aldrich), dehydrated with acetonitrile and air dried. Gel plugs were subjected to reducing (50 mM Tris (2-carboxyethyl) phosphine in $50 \mathrm{mM} \mathrm{TEAB}$ at $60{ }^{\circ} \mathrm{C}$ for $10 \mathrm{~min}$ ) and alkylation conditions (100 mM IAA in $50 \mathrm{mM}$ TEAB for $1 \mathrm{~h}$ in the dark) sequentially. Excised spots were dehydrated with acetonitrile and digested overnight at $37^{\circ} \mathrm{C}$ with $20 \mu \mathrm{g} / \mathrm{ml}$ proteomics grade porcine trypsin (Sigma-Aldrich).

nLC-ESI-MS/MS was performed using an Agilent 1100 Series HPLC coupled to an Agilent LC/MSD Trap XCT Plus Mass Spectrometer fitted with an HPLC Chip cube (Agilent, Palo Alto, CA, USA). The HPLC Chip comprised of a $40 \mathrm{nl}$ enrichment column and a $75 \mu \mathrm{m} \times 43 \mathrm{~mm}$ separation column both packed with Zorbax $300 S B-C_{18} 5 \mu \mathrm{m}$ particle size stationary phase. The LC method was controlled by Chemstation version B.01.03 (204). Samples were loaded onto the enrichment column in acetonitrile/formic acid (5\%/0.1\% v/v at $4 \mu \mathrm{l} / \mathrm{min})$. A $15 \mathrm{~min}$ linear gradient (flow rate $400 \mathrm{nl} / \mathrm{min}$ ) from 5 to $60 \%$ acetonitrile/ $0.1 \%$ formic acid $\mathrm{v} / \mathrm{v}$ was performed followed by a step from 60 to $80 \%$ acetonitrile/ $0.1 \%$ formic acid $\mathrm{v} / \mathrm{v}$ over $0.5 \mathrm{~min}$ and held for $1 \mathrm{~min}$ to elute any remaining protein from the column.

All MS/MS spectra were collected using data-dependent acquisition mode via MSD Trap Control Software version 5.3 build no. 11.0 (Bruker Daltonik GmbH, Bremen, Germany). After the acquisition of a full MS scan (m/z 300-1800 at $8100 \mathrm{~m} / \mathrm{z} / \mathrm{s}$ ) in the first scan event, the three most intense ions present above a threshold intensity of 10000 were subsequently isolated for fragmentation (MS/MS scan $\mathrm{m} / \mathrm{z}$ 100-2000 at $26000 \mathrm{~m} / \mathrm{z} / \mathrm{s})$. The collision energy for the MS/MS scan was ramped from 30 to $200 \%$ of $1.3 \mathrm{~V}$, for acquisition of the MS/MS scan and two spectra were averaged for each event. General instrument parameters were as follows: capillary 
voltage, $1950 \mathrm{~V}$; skimmer, $40 \mathrm{~V}$; capillary exit, $101.8 \mathrm{~V}$; trap drive, 74.4 ; dry gas, $4 \mathrm{l} / \mathrm{min}$; dry temperature $300{ }^{\circ} \mathrm{C}$.

The peptide sequences from the MS/MS spectra were identified by correlation with the peptide sequences from proteins present in the Swiss-Prot database (version 2011_06) using the MASCOT search algorithm (Matrix Science, London, UK). Typical MASCOT search parameters used were as follows: peptide mass tolerance, $\pm 1.2 \mathrm{Da}$; fragment mass tolerance, $\pm 0.6 \mathrm{Da}$; missed cleavages, 1; enzyme, trypsin; fixed modifications, cysteine modified by carbamidomethyl; variable modifications, oxidation and taxonomy, Homo sapiens.

\section{Two-dimensional PAGE}

The same samples were individually processed by the precipitation of the CVF proteins using the trichloroacetic acid precipitation method as described previously (Di Quinzio et al. 2007). Briefly, samples were incubated with equal volumes of $20 \%$ trichloroacetic acid and the precipitated protein pellet resolubilised in rehydration buffer. The protein concentration of each sample was determined using the Bradford Coomassie method. Fifteen micrograms of protein were subjected to first- and second-dimension separation in a similar method as described in the 2D-DIGE method. Gels were fixed and stained using SYPRO Ruby (Bio-Rad Laboratories) according to a previously published protocol (Di Quinzio et al. 2007). Gels were scanned at $100 \mathrm{~nm}$ resolution in a FX imager (Bio-Rad Laboratories). A match-set of all 15 gels was produced and spots of interest in the initial 2D-DIGE analysis were further investigated using PD Quest v7.3.1 Software (Bio-Rad Laboratories).

\section{D-PAGE statistical analysis}

Spot volumes calculated by PD Quest (area Xpixel density) were used to determine fold differences of matched spots compared between the two groups. The volumes of matched spots that showed significant differential expression from initial PD Quest analysis were exported to the Statistical Package for Social Sciences (SPSS v17, Chicago, IL, USA) for statistical analysis using the non-parametric Mann-Whitney $U$ test. A probability of $P<0.05$ was determined as being statistically significant.

\section{Western immunoblot}

An independent cohort of CVF samples (preterm PROM, $n=5$; gestation-matched controls, $n=10$ ) was selected for validation of candidate biomarkers using the inclusion/exclusion criteria as described previously. Twenty-five micrograms of CVF were resolved on a $15 \%$ Tris- $\mathrm{HCl}$ Criterion pre-cast gel (Bio-Rad Laboratories) under reducing conditions and proteins were transferred onto a polyvinylidene fluoride membrane. To confirm consistency of protein loading, we investigated the common 'house-keeping' proteins, actin, $\alpha$-tubulin and GAPDH in CVF, but found that these were not always expressed in all samples. There is no constitutively expressed protein in the CVF, as this is a secreted, non-sterile fluid. Therefore, to overcome this issue, the blot of individual samples in gel lanes was stained with SYPRO Ruby for 15 min (Bio-Rad Laboratories) and then imaged using the FX Imager (Bio-Rad Laboratories). Specific protein bands were then normalised against the total densitometry of each lane.

Blots were then blocked with 5\% skim milk in TBS-T $(0.025 \%$ Tween 20 in Tris-buffered saline) for $1 \mathrm{~h}$ at room temperature. Blots were probed with $1 \mu \mathrm{g} / \mathrm{ml}$ primary antibodies overnight at $4{ }^{\circ} \mathrm{C}$. The following primary monoclonal mouse antibodies were raised against human VDBP (clone 359501; R\&D Systems, Minneapolis, MN, USA), cystatin A (clone 224705; R\&D Systems) and thioredoxin (clone 2A7; Sigma-Aldrich). Polyclonal rabbit antibodies were raised against human annexin $A 3$ and FABP5 (Sigma-Aldrich). After three washes with TBS-T, blots were incubated with 1:2000 dilution of appropriate secondary antibody (goat anti-rabbit or goat anti-mouse IgG; Santa Cruz Biotechnology, Santa Cruz, CA, USA) for $1 \mathrm{~h}$ at room temperature. Blots were also incubated with biotinylated goat anti-human IL1 receptor antagonist $(0.2 \mu \mathrm{g} / \mathrm{ml}$; R\&D Systems) overnight at $4{ }^{\circ} \mathrm{C}$ followed by incubation with streptavidin-HRP ( $1: 200$ dilution; R\&D Systems) for $1 \mathrm{~h}$ at room temperature. All blots were washed three times with TBS-T and bands of interests were detected using electrochemiluminescence (Santa Cruz Biotechnology) and visualised on a Chemi-Doc Imager (Bio-Rad Laboratories). Volume densities of bands were determined using the Quantity One software (Bio-Rad Laboratories). Density values were exported into SPSS and analysed using the non-parametric Mann-Whitney $U$ test to determine statistical significance $(P<0.05)$.

\section{Supplementary data}

This is linked to the online version of the paper at http://dx.doi. org/10.1530/REP-12-0264.

\section{Declaration of interest}

The authors declare that there is no conflict of interest that could be perceived as prejudicing the impartiality of the research reported.

\section{Funding}

Funding was provided by the Medical Research Foundation for Women and Babies.

\section{Acknowledgements}

The authors would like to thank all participants involved in this study and the Mercy Hospital for Women medical and midwifery staff (Valerie Bryant and Mardi Reeves) for obtaining the CVF samples. The authors acknowledge Karen Oliva for technical assistance; Matthew Knight (Department of Primary Industries, Bundoora, Victoria, Australia) for use of the Typhoon 9400 scanner; Paul O'Donnell and Nick Williamson for their assistance with the nLC-ESI-MS/MS at the Bio21 Institute, Parkville, Victoria, Australia. Microbiological assessment was performed by the Microbiology Department, Austin Pathology, Austin Health, Heidelberg, Victoria, Australia. 


\section{References}

Ämmälä M, Nyman T, Salmi A \& Rutanen EM 1997 The interleukin-1 system in gestational tissues at term: effect of labour. Placenta $\mathbf{1 8}$ 717-723. (doi:10.1016/S0143-4004(97)90014-X)

Bastek JA, Sammel MD, Paré E, Srinivas SK, Posencheg MA \& Elovitz MA 2008 Adverse neonatal outcomes: examining the risks between preterm, late preterm, and term infants. American Journal of Obstetrics and Gynecology 199 e1-e8. (doi:10.1016/j.ajog.2008.08.002)

Bennaars-Eiden A, Higgins L, Hertzel AV, Kapphahn RJ, Ferrington DA \& Bernlohr DA 2002 Covalent modification of epithelial fatty acid-binding protein by 4-hydroxynonenal in vitro and in vivo. Evidence for a role in antioxidant biology. Journal of Biological Chemistry 277 50693-50702. (doi:10.1074/jbc.M209493200)

Biggio JR Jr, Ramsey PS, Cliver SP, Lyon MD, Goldenberg RL \& Wenstrom KD 2005 Midtrimester amniotic fluid matrix metalloproteinase-8 (MMP-8) levels above the 90th percentile are a marker for subsequent preterm premature rupture of membranes. American Journal of Obstetrics and Gynecology 192 109-113. (doi:10.1016/j. ajog.2004.06.103)

Cataltepe S, Gornstein ER, Schick C, Kamachi Y, Chatson K, Fries J, Silverman GA \& Upton MP 2000 Co-expression of the squamous cell carcinoma antigens 1 and 2 in normal adult human tissues and squamous cell carcinomas. Journal of Histochemistry and Cytochemistry $\mathbf{4 8}$ 113-122. (doi:10.1177/002215540004800112)

Crombach G, Scharl A, Vierbuchen M, Würz H \& Bolte A 1989 Detection of squamous cell carcinoma antigen in normal squamous epithelia and in squamous cell carcinomas of the uterine cervix. Cancer 63 1337-1342. (doi:10.1002/1097-0142(19890401)63:7 $\leq 1337:$ :AIDCNCR2820630719 23.0.CO;2-J)

Dasari S, Pereira L, Reddy AP, Michaels J-EA, Lu X, Jacob T, Thomas A, Rodland M, Roberts CT, Gravett MG et al. 2007 Comprehensive proteomic analysis of human cervical-vaginal fluid. Journal of Proteome Research 6 1258-1268. (doi:10.1021/pr0605419)

Davies ME \& Barrett AJ 1984 Immunolocalization of human cystatin in neutrophiles and lymphocytes. Histochemistry $\mathbf{8 0}$ 373-377. (doi:10.1007/BF00495420)

Di Quinzio MKW, Oliva K, Holdsworth SJ, Ayhan M, Walker SP, Rice GE, Georgiou HM \& Permezel M 2007 Proteomic analysis and characterisation of human cervico-vaginal fluid proteins. Australian \& New Zealand Journal of Obstetrics \& Gynaecology 47 9-15. (doi:10.1111/j. 1479-828X.2006.00671.x)

Di Quinzio MKW, Georgiou HM, Holdsworth-Carson SJ, Ayhan M, Heng YJ, Walker SP, Rice GE \& Permezel M 2008 Proteomic analysis of human cervico-vaginal fluid displays differential protein expression in association with labor onset at term. Journal of Proteome Research 7 1916-1921. (doi:10.1021/pr7006413)

Fortunato SJ, Menon R \& Lombardi SJ 1999 MMP/TIMP imbalance in amniotic fluid during PROM: an indirect support for endogenous pathway to membrane rupture. Journal of Perinatal Medicine 27 362-368. (doi:10.1515/JPM.1999.049)

Harper KD, McLeod JF, Kowalski MA \& Haddad JG 1987 Vitamin D binding protein sequesters monomeric actin in the circulation of the rat. Journal of Clinical Investigation 79 1365-1370. (doi:10.1172/JCI112963)

Helmig BR, Romero R, Espinoza J, Chaiworapongsa T, Bujold E, Gomez R, Ohlsson K \& Uldbjerg N 2002 Neutrophil elastase and secretory leukocyte protease inhibitor in prelabor rupture of membranes, parturition and intra-amniotic infection. Journal of Maternal-Fetal and Neonatal Medicine 12 237-246. (doi:10.1080/jmf.12.4.237.246)

Heng YJ, Di Quinzio MKW, Permezel M, Ayhan M, Rice GE \& Georgiou HM 2010a Temporal proteomic analysis of human cervicovaginal fluid with impending term labor. Journal of Proteome Research 9 1344-1350. (doi:10.1021/pr900892f)

Heng YJ, Di Quinzio MKW, Permezel M, Rice G \& Georgiou H $2010 b$ Temporal expression of antioxidants in human cervicovaginal fluid associated with spontaneous labor. Antioxidants \& Redox Signaling 13 951-957. (doi:10.1089/ars.2010.3122)

Heng YJ, Di Quinzio MKW, Permezel M, Rice GE \& Georgiou HM 2011 Cystatin A protease inhibitor and cysteine proteases in human cervicovaginal fluid in term pregnancy and labor. American Journal of Obstetrics and Gynecology 204 254.e1-254.e7. (doi:10.1016/j.ajog. 2010.10.912)
Japelj B, Waltho JP \& Jerala R 2004 Comparison of backbone dynamics of monomeric and domain-swapped stefin A. Proteins 54 500-512. (doi:10.1002/prot.10624)

Klein LL, Jonscher KR, Heerwagen MJ, Gibbs RS \& McManaman JL 2008 Shotgun proteomic analysis of vaginal fluid from women in late pregnancy. Reproductive Sciences 15 263-273. (doi:10.1177/1933 719107311189)

Le Cabec V \& Maridonneau-Parini I 1994 Annexin 3 is associated with cytoplasmic granules in neutrophils and monocytes and translocates to the plasma membrane in activated cells. Biochemical Journal 303 481-487.

Longini M, Perrone S, Vezzosi P, Marzocchi B, Kenanidis A, Centini G, Rosignoli L \& Buonocore G 2007 Association between oxidative stress in pregnancy and preterm premature rupture of membranes. Clinical Biochemistry 40 793-797. (doi:10.1016/j.clinbiochem. 2007.03.004)

Lysell J, Stjernholm Vladic Y, Ciarlo N, Holmgren A \& Sahlin L 2003 Immunohistochemical determination of thioredoxin and glutaredoxin distribution in the human cervix, and possible relation to cervical ripening. Gynecological Endocrinology 17 303-310. (doi:10.1080/gye. 17.4.303.310)

Malyak M, Smith MF Jr, Abel AA \& Arend WP 1994 Peripheral blood neutrophil production of interleukin-1 receptor antagonist and interleukin-1ß. Journal of Clinical Immunology 14 20-30. (doi:10.1007/ BF01541172)

Martin JR, Craven CJ \& Jerala R 1995 The three-dimensional solution structure of human stefin A. Journal of Molecular Biology 246 331-343. (doi:10.1006/jmbi.1994.0088)

McLaren J, Malak TM \& Bell SC 1999 Structural characteristics of term human fetal membranes prior to labour: identification of an area of altered morphology overlying the cervix. Human Reproduction $\mathbf{1 4}$ 237-241. (doi:10.1093/humrep/14.1.237)

McLeod JF, Kowalski MA \& Haddad JG 1989 Interactions among serum vitamin D binding protein, monomeric actin, profilin, and profilactin. Journal of Biological Chemistry 264 1260-1267.

Mercer BM 2003 Preterm premature rupture of the membranes. Obstetrics and Gynecology 101 178-193. (doi:10.1016/S0029-7844 (02)02366-9)

Moore RM, Mansour JM, Redline RW, Mercer BM \& Moore JJ 2006 The physiology of fetal membrane rupture: insight gained from the determination of physical properties. Placenta 27 1037-1051. (doi:10.1016/j.placenta.2006.01.002)

Nakamura H, Nakamura K \& Yodoi J 1997 Redox regulation of cellular activation. Annual Review of Immunology 15 351-369. (doi:10.1146/ annurev.immunol.15.1.351)

Pastore A, Federici G, Bertini E \& Piemonte F 2003 Analysis of glutathione: implication in redox and detoxification. Clinica Chimica Acta 333 19-39. (doi:10.1016/S0009-8981(03)00200-6)

Pereira L, Reddy AP, Jacob T, Thomas A, Schneider KA, Dasari S, Lapidus JA, Lu X, Rodland M, Roberts CT et al. 2007 Identification of novel protein biomarkers of preterm birth in human cervical-vaginal fluid. Journal of Proteome Research 6 1269-1276. (doi:10.1021/ pr0605421)

Perkins AV, Di Trapani G, McKayb MS \& Clarke FM 1995 Immunocytochemical localization of thioredoxin in human trophoblast and decidua. Placenta 16 635-642. (doi:10.1016/0143-4004(95)90032-2)

Piquette CA, Robinson-Hill R \& Webster RO 1994 Human monocyte chemotaxis to complement-derived chemotaxins is enhanced by Gc-globulin. Journal of Leukocyte Biology 55 349-354.

Reddy VY, Zhang QY \& Weiss SJ 1995 Pericellular mobilization of the tissue-destructive cysteine proteinases, cathepsins B, L, and S, by human monocyte-derived macrophages. PNAS 92 3849-3853. (doi:10.1073/ pnas.92.9.3849)

Reti NG, Lappas M, Riley C, Wlodek ME, Permezel M, Walker S \& Rice GE 2007 Why do membranes rupture at term? Evidence of increased cellular apoptosis in the supracervical fetal membranes American Journal of Obstetrics and Gynecology 196 484.e1-484.e10. (doi:10.1016/j.ajog. 2007.01.021)

Rosen T, Kuczynski E, O'Neill LM, Funai EF \& Lockwood CJ 2001 Plasma levels of thrombin-antithrombin complexes predict preterm premature rupture of the fetal membranes. Journal of Maternal-Fetal Medicine $\mathbf{1 0}$ 297-300. (doi:10.1080/jmf.10.5.297.300) 
Saitoh M, Nishitoh H, Fujii M, Takeda K, Tobiume K, Sawada Y, Kawabata M, Miyazono K \& Ichijo H 1998 Mammalian thioredoxin is a direct inhibitor of apoptosis signal-regulating kinase (ASK) 1. EMBO Journal 17 2596-2606. (doi:10.1093/emboj/17.9.2596)

Savitz DA, Blackmore CA \& Thorp JM 1991 Epidemiologic characteristics of preterm delivery: etiologic heterogeneity. American Journal of Obstetrics and Gynecology 164 467-471.

Schick C, Pemberton PA, Shi G-P, Kamachi Y, Çataltepe S, Bartuski AJ, Gornstein ER, Brömme D, Chapman HA \& Silverman GA 1998 Cross-class inhibition of the cysteine proteinases cathepsins $\mathrm{K}, \mathrm{L}$, and S by the serpin squamous cell carcinoma antigen 1: a kinetic analysis. Biochemistry 37 5258-5266. (doi:10.1021/bi972521d)

Shah SJ, Yu KH, Sangar V, Parry SI \& Blair IA 2009 Identification and quantification of preterm birth biomarkers in human cervicovaginal fluid by liquid chromatography/tandem mass spectrometry. Journal of Proteome Research 8 2407-2417. (doi:10.1021/pr8010342)

Shaw JLV, Smith CR \& Diamandis EP 2007 Proteomic analysis of human cervico-vaginal fluid. Journal of Proteome Research 6 2859-2865. (doi:10.1021/pr0701658)

Siegenthaler G, Hotz R, Chatellardgruaz D, Jaconi S \& Saurat JH 1993 Characterization and expression of a novel human fatty acid-binding protein: the epidermal type (E-FABP). Biochemical and Biophysical Research Communications 190 482-487. (doi:10.1006/bbrc.1993. 1073)

Simhan HN \& Canavan TP 2005 Preterm premature rupture of membranes: diagnosis, evaluation and management strategies. BJOG : an International Journal of Obstetrics and Gynaecology 112 32-37. (doi:10.1111/j.1471-0528.2005.00582.x)

Sugimori T, Cooley J, Hoidal JR \& Remold-O'Donnell E 1995 Inhibitory properties of recombinant human monocyte/neutrophil elastase inhibitor. American Journal of Respiratory Cell and Molecular Biology 13 314-322.

Tait JF, Sakata M, McMullen BA, Miao CH, Funakoshi T, Hendrickson LE \& Fujikawa K 1988 Placental anticoagulant proteins: isolation and comparative characterization of four members of the lipocortin family. Biochemistry 27 6268-6276. (doi:10.1021/bi00417a011)

Tang L, De Seta F, Odreman F, Venge P, Piva C, Guaschino S \& Garcia RC 2007 Proteomic analysis of human cervical-vaginal fluids. Journal of Proteome Research 6 2874-2883. (doi:10.1021/pr0700899)

Turk V \& Bode W 1991 The cystatins: protein inhibitors of cysteine proteinases. FEBS Letters 285 213-219. (doi:10.1016/0014-5793(91) 80804-C)
Venkataraman N, Cole AL, Svoboda P, Pohl J \& Cole AM 2005 Cationic polypeptides are required for anti-HIV-1 activity of human vaginal fluid. Journal of Immunology 175 7560-7567.

Wang H, Parry S, Macones G, Sammel M, Kuivaniemi H, Tromp G, Argyopoulos G, Halder I, Schriver M, Romero R et al. 2006 A functional SNP in the promoter of the SERPINH1 gene increases risk of preterm premature rupture of membranes in African Americans. PNAS 103 13463-13467. (doi:10.1073/pnas.0603676103)

Wang H, Sammel MD, Tromp G, Gotsch F, Halder I, Schriver MD, Romero R \& Strauss JF III 2008 A 12-bp deletion in the $5^{\prime}$-flanking region of the SERPINH1 gene affects promoter activity and protects against preterm premature rupture of membranes in African Americans. Human Mutation 29 332. (doi:10.1002/humu.9522)

Watari M, Watari H, DiSanto ME, Chacko S, Shi G-P \& Strauss JF III 1999 Pro-inflammatory cytokines induce expression of matrix-metabolizing enzymes in human cervical smooth muscle cells. American Journal of Pathology 154 1755-1762. (doi:10.1016/S0002-9440(10)65431-4)

White P \& Cooke N 2000 The mutifunctional properties and characteristics of vitamin D-binding protein. Trends in Endocrinology and Metabolism 11 300-307. (doi:10.1016/S1043-2760(00)00317-9)

Yamamoto N \& Homma S 1991 Vitamin D3 binding protein (groupspecific component) is a precursor for the macrophage-activating signal factor from lysophosphatidylcholine-treated lymphocytes. PNAS $\mathbf{8 8}$ 8539-8543. (doi:10.1073/pnas.88.19.8539)

Yamamoto N \& Kumashiro R 1993 Conversion of vitamin $D_{3}$ binding protein (group-specific component) to a macrophage activating factor by the stepwise action of $\beta$-galactosidase of B cells and sialdase of $\mathrm{T}$ cells. Journal of Immunology $1512794-2802$.

Zegels G, Van Raemdonck G, Coen E, Tjalma W \& Van Ostade X 2009 Comprehensive proteomic analysis of human cervical-vaginal fluid using colposcopy samples. Proteome Science 7 17. (doi:10.1186/14775956-7-17)

Zegels G, Van Raemdonck GAA, Tjalma WAA \& Van Ostade XWM 2010 Use of cervicovaginal fluid for the identification of biomarkers for pathologies of the female genital tract. Proteome Science 863 . (doi:10.1186/1477-5956-8-63)

Received 12 July 2012

First decision 20 September 2012

Revised manuscript received 25 October 2012

Accepted 22 November 2012 